\title{
Suppression of a rewarded response by punishment as a function of reinforcement schedules'
}

\author{
M. VOGEL-SPROTT \\ UNIVERSITY OF WATERLOO
}

Four groups of 15 human $S$ s were trained on a buttonpressing response for money. During acquisition, two groups received $100 \%$ continuous reward (C) for the correct response and two groups received 50\% intermittent reward (I). These reward schedules were maintained when punishment of the response subsequently was introduced. One $\mathrm{C}$ and one $\mathrm{I}$ group received $100 \%$ continuous punishment (CP) and the two remaining groups received $50 \%$ intermittent punishment (IP). Groups receiving $\mathrm{CP}$ suppressed the response in fewer trials than those receiving IP ( $p<.005)$, but suppression rates of the $C$ groups did not differ from the I groups.

Research on human Ss (Banks \& Vogel-Sprott, 1965) recently examined the suppressing effect of punishment (electric shock) on an immediately rewarded (money) response. The study adopted a $100 \%$ reinforcement schedule for both reinforcers and manipulated only the time delay in punishment. A gradient effect of punishment on response suppression was obtained as a function of punishment delay. The present experiment continues the investigation of this type of conflict situation, holding punishment delay constant at zero and manipulating only the schedule of the reinforcers.

Method

Sixty undergraduate students at University of Waterloo were allocated randomly into four groups of $15 \mathrm{Ss}$ each.

The apparatus consisted of a three-button responsepanel, a penny reward dispenser and an 850-v shock generator with leads to finger electrodes. The equipment was connected so that one particular sequence of three presses on the button (e.g., 3-1-2) could activate both the reward dispenser and the shock generator for immediate delivery of a penny and shock (1.75 ma for $.10 \mathrm{sec}$ ). The schedule of these reinforcers could be independently programmed by the apparatus so that a consistent $(100 \%)$ or an intermittent (50\%) occurrence of reward could be combined with either a $100 \%$ or $50 \%$ punishment schedule. The reward dispenser and response-panel were placed on the desk at which $S$ sat. He was informed that he would have a number of buttonpressing trials for pennies which he could keep. Trials would be signalled by the offset of S's response-panel lights. On each trial, he was free to press the three buttons in any order he wished, provided that no button was pressed twice in the same trial. (This restricted the number of possible sequences to six.) Although the penny reward was administered only for one of these sequences, $\mathrm{S}$ was not given this information. The $\mathrm{E}$ then attached finger electrodes to $S$ 's nondominant hand with the commrnt that he may sometimes receive a slight electric shock, and if he found a way of working which allowed him to avoid shock he should do so.

The $\mathrm{S}$ then began button-pressing trials which were presented at $15-\mathrm{sec}$. intervals. Trials continued until the "correct" response (i.e., the one rewarded sequence) was displayed on five consecutive trials. This learning criterion is identical to that previously employed in other studies (cf., Banks \& Vogel-Sprott, 1965). Two groups of Ss received this training under $100 \%$ continuous reward (C) and the remaining two groups were trained under a 50\% intermittent reward (I) schedule. The training of the two I groups was based on a predetermined schedule devised so that 10 rewards were administered for a set of 20 correct responses. The first occurrence of the correct response received reward, and the response received no more than two consecutive rewards. This schedule was followed for each $S$ and was repeated until S's performance met criterion.

Twenty punishment trials were administered immediately after the response met criterion. All groups continued on their respective reward schedules during these punishment trials, but one $\mathrm{C}$ and one I group received immediate continuous punishment (CP) of the correct response each time it was displayed. These two groups henceforth are identified by their combined reinforcement schedules, as CC and IC respectively. A 50\% intermittent punishment (IP) schedule was administered to the remaining two groups, designated as CI and II. The IP schedule was devised so that the first correct response occurring on punishment trials received shock, and this response received no more than two consecutive punishments. Thus, if $\mathrm{S}$ displayed the correct response on all 20 punishment trials, he would receive a maximum of 10 shocks.

\section{Results and Discussion}

The mean number of punished responses in each group is as follows: $\mathrm{CC}=8.47\left(\sigma^{2}=31.9\right), \mathrm{IC}=8.93\left(\sigma^{2}=\right.$ 23.79), $\mathrm{CI}=14.47 \quad\left(\sigma^{2}=20.78\right), \mathrm{II}=11.67 \quad\left(\sigma^{2}=27.42\right)$.

A two-way variance analysis of punished response scores indicates no significant interaction effects between reward and punishment schedules $(\mathrm{F}=2.2$, $\mathrm{df}=$ $1 / 56, p>.10)$ and no significant main effect of reward schedule $(F=0.5, d f=1 / 56, p>.10)$. A strong main effect of punishment is observed ( $F=9.9, \mathrm{df}=1 / 56, \mathrm{p}<.005)$ and the group means above indicate that fewer punished responses occur under CP than under IP. Since the former schedule administered more noxious stimulation for the response, its superior suppressing effect is not surprising, and is in line with evidence of greater sup- 
pression under CP than under IP of a response undergoing extinction (Estes, 1944).

The particular reward schedule employed in establishing the response, and maintained during punishment, failed to influence responding during suppression trials. This finding cannot be attributed merely to an ineffective manipulation of reward schedule, for the data on acquisition trials reveal systematic differences between $\mathrm{C}$ and $\mathrm{I}$ training. $\mathrm{C}$ groups required an average of 8.5 trials ( $\sigma^{2}=18.89$ ) to meet the learning criterion whereas I groups required a mean of $36.7\left(\sigma^{2}=706.08\right)$ acquisition trials $(t=4.77, d f=58, p<.01)$. The failure to observe an effect of reward schedule during punishment trials contrasts with the differential effect of reward schedules typically observed during extinction trials. Unfortunately, extinction trials were not involved in the present study since the response continued to receive reward during the administration of punishment. Thus, the possible differential effects of $\mathrm{C}$ and $\mathrm{I}$ reward schedules on a response punished during extinction in this situation remain to be determined.

This study might be considered to suggest that the reward schedule employed in training a response and maintained when punishment is introduced may be unrelated to the ease of suppressing this response. Further research, however, is required to assess the generality of such a conclusion for many variables related to reinforcement remain to be manipulated in this situation. For example, the response in $\mathrm{C}$ and I groups was trained to the same asymptote (i.e., five consecutive responses) prior to the onset of punishment. This learning criterion is of interest in its own right, since humans encounter many situations in which punishment of a response begins only after it is well learned. It should be noted however that if acquisition trials continue until performance under intermittent reward equals that displayed under continuous reward, groups under these two schedules will differ systematically in trials. This training procedure differs from that customarily employed in other studies demonstrating an effect of reward schedules (cf., Brown \& Wagner, 1964) for such research usually holds trials constant under each schedule and allows response strength to vary. The failure to obtain an effect of reward schedules in the present study thus might be attributed to the particular acquisition criterion employed. A series of studies which systematically manipulate some of the response parameters (e.g., acquisition criterion, reward and punishment values) thus are required both to assess the generality of the obtained evidence and to assist in clarifying the apparent discrepancy between this study and animal data (Brown \& Wagner, 1964) which indicates that intermittent reward training may mitigate the suppressing effect of punishment.

\section{References}

Banks, R. K., \& Vogel-Sprott, M. D. The effect of delayed punishment on an immediately rewarded response in humans. J. exp. Psychol., 1965, 70, 357-359.

Brown, R. T., \& Wagner, A. R. Resistance to punishment and extinction following training with shock or nonreinforcement. $J$. exp. Psychol., 1964, 68, 503-507.

Estes, W. K. An experimental study of punishment. Psychol. Monogr., 1944, (Whole No, 263), Exp. E.

\section{Note}

1.. This research was supported by a N.R.C. Grant APA-93. The assistance of Mr. A. Shapiro is gratefully acknowledged. 